\title{
Clinical outcomes of gastrointestinal brain metastases treated with radiotherapy
}

\author{
Samrat M. Sanghvi ${ }^{*}$, Jonathan W. Lischalk², Ling Cai ${ }^{3}$, Sean Collins ${ }^{2}$, Mani Nair ${ }^{4}$, Brain Collins ${ }^{2}$ and Keith Unger ${ }^{2}$
}

\begin{abstract}
Background: Brain metastases of gastrointestinal origin are a rare occurrence. Radiation therapy (RT) in the form of stereotactic radiosurgery (SRS) or whole brain radiation therapy (WBRT) is an effective established treatment modality in either the definitive or adjuvant setting. The aim of this study is to assess the long-term clinical outcomes of patients with gastrointestinal (GI) brain metastases treated with SRS or WBRT.

Methods: In this single institutional retrospective review, we detail the outcomes of patients diagnosed with metastatic brain tumors from an adenocarcinoma gastrointestinal primary. Patients were treated using stereotactic radiosurgery or whole brain radiation therapy. Initial site control (defined as lesions visualized on imaging at time of treatment), new site control (defined as new intracranial lesions visualized on follow-up imaging), and overall survival were calculated using the Kaplan-Meier method.
\end{abstract}

Results: Thirty-three patients were treated from August 2008 to December 2015. Primary malignancy locations were as follows: 18 colon, 6 esophagus, 4 rectum, 5 other. Median total dose delivered was 25 Gy (18-35 Gy) in a median of 4 fractions for SRS and 30 Gy (10.8-40 Gy) in 10 fractions for WBRT. Crude initial site control at last radiographic follow-up was $64.3 \%$ after SRS and $41.7 \%$ after WBRT. Eleven of the 28 brain lesions (39.3\%) treated with SRS had resection of the SRS-treated lesion prior to radiation therapy. Five of the twelve patients (41.7\%) undergoing WBRT underwent cranial resection prior to radiation therapy. Crude new site control at last radiographic follow-up was $46.4 \%$ after SRS and $83.3 \%$ after WBRT. Kaplan-Meier analysis of overall survival did not show any statistically significant difference between WBRT and SRS $(p=0.424)$. Median overall survival for SRS patients was 5.2 months (0.5-57.5) and for WBRT patients 4.4 months (0-15). Kaplan-Meier analysis of new site control was significantly improved with WBRT versus SRS $(p=0.017)$. Total dose, treatment with WBRT, and active extracranial disease were statistically significant on multivariate analysis for new site control $(p<0.05)$.

Conclusions: Survival and intracranial disease control are poor following RT for brain metastases from Gl primaries. In this small series, outcomes are worse than published series for other primary malignancies metastatic to the brain and further research into methods of local control improvement is warranted. Future studies should explore the utility of dose escalation or radiosensitization in this patient population.

Keywords: Radiosurgery, Gastrointestinal neoplasms, Brain neoplasm, Metastases

\section{Introduction}

Gastrointestinal (GI) malignancies represent a significant burden of disease in the United States with colorectal cancer representing nearly $10 \%$ of all new cancer diagnoses and ranking as the second leading cause of cancer related death [1]. These sites rarely metastasize to the brain with reported rates at less than 9\% [2-6].

\footnotetext{
*Correspondence: SMS382@georgetown.edu

'Georgetown University School of Medicine, Medical Dental Building, 3900

Reservoir Road, N.W, Washington DC 20057, USA

Full list of author information is available at the end of the article
}

Nevertheless, given the prevalence of GI malignancies, brain metastases from these primaries still represent 4-8\% of all diagnosed brain metastases [3, 7-9]. Moreover, their incidence is increasing possibly due to longer survival seen in patients with colorectal primaries as a result of improvements in systemic therapy and earlier diagnosis $[10,11]$. Additionally, wider utilization of brain MRIs has contributed to an increased diagnosis of previously occult brain metastases [12]. 
Historically, whole brain radiation therapy (WBRT) has been the standard of care for brain metastases given the lack of accurate targeting coupled with the concern for microscopic disease elsewhere in the brain [13]. With improving targeting technology however, stereotactic radiosurgery (SRS) has emerged as an alternative treatment modality for many cases of metastatic disease to the brain. As compared to WBRT, SRS has the potential to be completed in less time, achieve increased efficacy against radioresistant tumors, increase the likelihood of reirradiation, decrease hair loss, and mitigate neurocognitive side effects [14-17].

Literature documenting clinical outcomes of patients with brain metastasis from GI primaries treated with radiation therapy is scarce and focuses mainly on outcomes following Gamma Knife radiosurgery (GKS) and not LINAC-based techniques, which are becoming a more prevalent modality of SRS. The purpose of this retrospective review is to explore the clinical outcomes, local and distant intracranial control rates, and prognostic factors associated with GI brain metastasis treated with WBRT and SRS.

\section{Materials and methods \\ Patient eligibility}

The local Institutional Review Board approved this retrospective analysis of an established departmental treatment approach. The analysis was conducted utilizing the medical records of patients diagnosed with a metastatic brain tumor from a primary gastrointestinal malignancy between 2008 and 2015. Inclusion criteria was as follows: (1) primary adenocarcinoma malignancy originating from the colon, rectum, pancreas, esophagus, rectosigmoid, duodenum, or stomach, (2) histologic confirmation of primary gastrointestinal malignancy, and (3) brain metastasis confirmed by computed tomography (CT) scan or by magnetic resonance imaging (MRI). Exclusion criteria were as follows: (1) prior history of malignancy within the last 5 years, and (2) lack of radiographic follow-up (specifically for intracranial control analyses). Patients without radiographic follow-up were included in the overall survival analysis. Lesions that underwent surgical resection prior to radiotherapy were included. Sites of distant extracranial disease were identified utilizing positron emission tomography (PET), skeletal scintigraphy, CT, and/or MR imaging as deemed necessary by medical oncology performed within 2 months of original intracranial metastatic diagnosis. If no imaging modality was available within 2 months, distant extracranial disease was determined by clinical exam of the primary medical oncologist in conjunction with prior imaging at the time of development of brain metastases. Twelve patients had PET scans for extracranial disease determination, fifteen utilized a CT of the thorax, abdomen, and pelvis with contrast in conjunction with prior PET scans, five utilized alternate imaging modalities in conjunction with older imaging, and one patient was staged clinically with utilization of alternate imaging modalities. Extracranial disease status was determined by the official radiology report [18].

\section{Treatment planning and delivery}

Patients were treated in the supine position with a custom aquaplast mask for reproducible patient setup. A fine-cut contrast enhanced treatment planning $\mathrm{CT}$ scan of the brain was obtained for each patient in the supine treatment position using a GE LightSpeed RT16. SRS treatment planning and delivery was done on the CyberKnife Robotic SRS system as detailed previously [19]. WBRT was performed with $6 \times$ photons delivered using opposed laterals with custom MLC blocking and a thermoplastic mask. The radiation therapy modality (SRS vs. WBRT) was selected at the discretion of the treating radiation oncologist, based on the number of lesions, lesion size, location of the lesions, and the patient's performance status. The radiation dose for WBRT and SRS was selected at the discretion of the treating radiation oncologist; SRS dose and fractionation was primarily based on target volume and location within or adjacent to an eloquent area of the cortex.

\section{Follow up}

Patients were followed with MRI or CT imaging at three to six month intervals according to routine institutional practice. Initial site control was defined as no progression in size of the initial tumor. Progression was defined based on increased tumor size, T1 post contrast enhancement, mass effect, fluid-attenuated inversion recovery, and/or vasogenic edema. For WBRT, initial site was defined as all tumors seen on brain imaging at time of treatment, and for SRS was specified as the treated tumor. New intracranial lesions were defined as new brain masses of any size noted on follow-up imaging. New site control was defined as no new development of intracranial lesions. If new intracranial lesions were noted, this was defined as a failure of new site control. We were unable to determine the cause of death from the available medical records in a sufficient number of patients.

\section{Statistical analysis}

Statistical analysis was performed with the $\mathrm{R}$ statistical software, version 3.2.1 ( $\mathrm{R}$ Foundation for Statistical Computing, http://www.r-project.org, Vienna, Austria). Baseline patient and tumor values (age, treatment length, dose, etc.) were analyzed for descriptive 
characteristics (mean, median, etc.). The follow-up duration was defined as the time from treatment completion to the last date of imaging follow-up. Tumors were censored at the date of last radiographic follow-up for initial and new site control analysis. Kaplan-Meier estimates were used for the calculation of overall survival (OS), initial site control, and new site control. The logrank test and the Cox proportional hazards model were used to analyze the factors affecting overall survival, initial site control, and new site control. A $p$ value $<0.05$ was regarded as statistically significant.

\section{Results}

\section{Patient characteristics}

Thirty-three patients were treated from August 2008 to December 2015. The median age was 62 years $(29$ to 83 years). The majority of patients were male $(66 \%)$ and median pretreatment ECOG performance status was 1 (0 to 3). Distribution of primary tumor location was as follows: 18 colon, 6 esophagus, 4 rectum, 2 gastric, 1 rectosigmoid, 1 duodenal, and 1 pancreas. The majority of patients had active extracranial disease at the time of diagnosis of brain metastases (87.9\%). Twenty patients had liver metastases $(60.6 \%)$ and 20 patients had lung metastases $(60.6 \%)$, with 16 of those patients having both liver and lung metastases (48.5\%) at the time of presentation with brain metastases. Median number of brain tumors at initial presentation was two (1 to 5). Summary patient characteristics are shown in Table 1. Two patients had brain metastases at initial primary site cancer diagnosis. Median time between initial cancer diagnosis and brain metastasis diagnosis was 18.1 months. Of those with radiographic follow-up, 12 patients were treated with WBRT and 16 patients were treated with SRS. Eleven of the 28 brain lesions (39.3\%) treated with SRS had resection of the SRS-treated lesion prior to radiation therapy. Five of the twelve patients $(41.7 \%)$ undergoing WBRT underwent cranial resection prior to

Table 1 Patient characteristics

\begin{tabular}{|c|c|c|c|c|}
\hline Treatment & $\begin{array}{l}\text { WBRT } \\
(n=12)\end{array}$ & $\begin{array}{l}\text { SRS } \\
(n=21)\end{array}$ & $\begin{array}{l}\text { Total } \\
(n=33)\end{array}$ & $P$-value \\
\hline Median age (range) & $62(41 \sim 76)$ & $62(29 \sim 83)$ & $62(29 \sim 83)$ & 0.963 \\
\hline Median interval between cancer diagnosis and brain metastases (range) & $422.5(0 \sim 2155)$ & $905(22 \sim 1668)$ & $542(0 \sim 2155)$ & 0.779 \\
\hline \multicolumn{5}{|l|}{ Brain metastases at initial presentation $[N(\%)]$} \\
\hline Yes & $2(100)$ & $0(0)$ & 2 & \multirow[t]{2}{*}{0.125} \\
\hline No & $10(32)$ & $21(68)$ & 31 & \\
\hline \multicolumn{5}{|l|}{ Intracranial surgery prior to radiation therapy $[N(\%)]$} \\
\hline No & $7(50)$ & $7(50)$ & 14 & \multirow[t]{2}{*}{0.273} \\
\hline Yes & $5(26)$ & $14(74)$ & 19 & \\
\hline \multicolumn{5}{|l|}{ Primary site $[n(\%)]$} \\
\hline Colon & $4(24)$ & $13(76)$ & 17 & \multirow[t]{4}{*}{0.247} \\
\hline Esophagus & $3(50)$ & $3(50)$ & 6 & \\
\hline Other & $4(67)$ & $2(33)$ & 6 & \\
\hline Rectum & $1(25)$ & $3(75)$ & 4 & \\
\hline \multicolumn{5}{|l|}{ BMs $[n(\%)]$} \\
\hline$\leq 2$ & $4(17)$ & $19(83)$ & 23 & \multirow[t]{2}{*}{$0.001^{*}$} \\
\hline$>2$ & $8(80)$ & $2(20)$ & 10 & \\
\hline \multicolumn{5}{|l|}{ ECOG [N (\%)] } \\
\hline Unknown & $0(0)$ & $1(100)$ & 1 & \multirow[t]{3}{*}{0.273} \\
\hline$\leq 1$ & $8(50)$ & $8(50)$ & 16 & \\
\hline$>1$ & $4(25)$ & $12(75)$ & 16 & \\
\hline \multicolumn{5}{|l|}{ RPA class [N (\%)] } \\
\hline Unknown & $0(0)$ & $1(100)$ & 1 & \multirow[t]{3}{*}{0.144} \\
\hline | and || & $8(53)$ & $7(47)$ & 15 & \\
\hline III & $4(24)$ & $13(76)$ & 17 & \\
\hline
\end{tabular}

Abbreviations: WBRT whole brain radiation therapy, SRS stereotactic radiosurgery, BMs brain metastases, ECOG Eastern Cooperative Oncology Group, RPA recursive partitioning analysis

Statistically significant results indicated by * 
radiation therapy. Median time between surgery and radiation therapy was 28 days with a mean of 46.33 days. One lesion was treated with SRS followed by planned WBRT.

\section{Treatment characteristics}

Table 2 shows baseline tumor and treatment characteristics. Median total dose delivered was 25 Gy (18-35 Gy) for SRS and 30 Gy (10.8-40 Gy) for WBRT. Median number of fractions for SRS was four (1-4) and for WBRT was ten (6-20). Median gross tumor volume (GTV) treated with SRS was $4.6 \mathrm{cc}(0.11-95.19 \mathrm{cc})$ and mean GTV was $12.08 \mathrm{cc}$. Biologically equivalent dose (BED) was calculated utilizing the linear-quadratic approach with an alpha/beta value of ten. For SRS the median BED was 48 Gy (37.5 to $93.6 \mathrm{~Gy}$ ), and for WBRT it was 39 Gy (12.74-48 Gy).

Overall, five patients received SRS salvage after initial WBRT and six patients received WBRT salvage after initial SRS. The following lesions were excluded from the new site control analysis for the SRS group: (1) one patient with planned WBRT after SRS, and (2) two patients with previous WBRT within 6 months. Figure 1 shows patient attrition through the study. The radiographic follow-up for the initial treated lesion was one scan for ten patients, two scans for five patients, three scans for four patients, and equal to or greater than four scans for four patients.

\section{Outcomes}

Crude initial site control (also referred to as local control for SRS) at last radiographic follow-up was $64.3 \%$ after SRS and $41.7 \%$ after WBRT. Crude new site control (also referred to as distant intracranial control for SRS) defined as no new lesions seen within the brain at last radiographic follow-up were $46.4 \%$ after SRS and $83.3 \%$ after WBRT. Median radiographic follow-up for SRS was 3.9 months (0.7-57.3) and for WBRT was 4.1 months (0.7-53.8). Kaplan-Meier analysis for initial site control and new site control is shown in Fig. 2a and b respectively. Kaplan-Meier analysis for overall survival is shown in Fig. 2c. For SRS, the actuarial 6-month local control was $47 \%$, the actuarial 6 -month new intracranial lesion control was $21 \%$, and the actuarial 6-month overall survival was $45 \%$. Median overall survival for SRS patients was 5.2 months (0.5-57.5) and for WBRT patients 4.4 months $(0-15)$. There was no statistically significant difference between the two treatments in regards to initial site control and overall survival as determined by Kaplan-Meier analysis ( $p=0.323$ and 0.424 , respectively). There was a significant difference between the two treatments in regards to new site control, which was found to be superior in the WBRT group ( $p=0.017)$.

In univariate analysis (UVA) for overall survival, the following variables were analyzed: age, total dose, treatment length, interval between initial cancer diagnosis and brain metastasis diagnosis, surgery prior to RT for largest lesion, presence of extracranial disease, number of brain metastases ( $\leq 2$ vs $>2$ ), ECOG ( $\leq 1$ vs $>1$ ), RPA class, and site of primary disease. None of these variables were statistically significant (results not shown). In addition, none of the variables analyzed on multivariate analysis (MVA) of overall survival were found to be statistically significant (results shown in Table 3).

In UVA for initial site control and new intracranial lesion control, the following variables were analyzed: surgical resection prior to RT, number of brain metastases, total dose, tumor volume, and tumor coverage. None of these variables were found to be statistically significant (results not shown). On MVA for initial site control, none of the analyzed variables were found to be statistically significant (results shown in Table 3). On MVA for new site control, the following variables were found to be statistically significant: total dose, treatment with WBRT, and having active extra cranial disease (results displayed in Table 3).

\section{Discussion}

Brain metastases are generally considered a late event for patients with gastrointestinal primaries with reported rates at presentation of only $0.2 \%$ [5]. Hence, patients rarely present with only metastases to the brain and more commonly develop liver or lung metastases prior

Table 2 Tumor and treatment characteristics

\begin{tabular}{lll}
\hline & SRS $(n=28)$ & WBRT $(n=12)$ \\
\hline Median tumor volume in cubic centimeters (range) & $4.6(0.11-95.19)$ & - \\
Median tumor coverage (range) & $99.41(97.9-100)$ & - \\
Median Dose per fraction in cGy (range) & $750(500-2600)$ & $300(180-300)$ \\
Median fractions (range) & $4(1-5)$ & $10(6-20)$ \\
Median total dose in cGy (range) & $2500(1800-3500)$ & $3000(1080-4000)$ \\
Median biologically equivalent dose in Gy (range) & $48(37.5-93.6)$ & $39(12.74-48)$ \\
Median length of treatment in days (range) & $4(1-12)$ & $15(14-71)$ \\
Prior WBRT & 2 & 0 \\
\hline
\end{tabular}

Abbreviations: SRS stereotactic radiosurgery, WBRT whole brain radiation therapy, Gy gray 


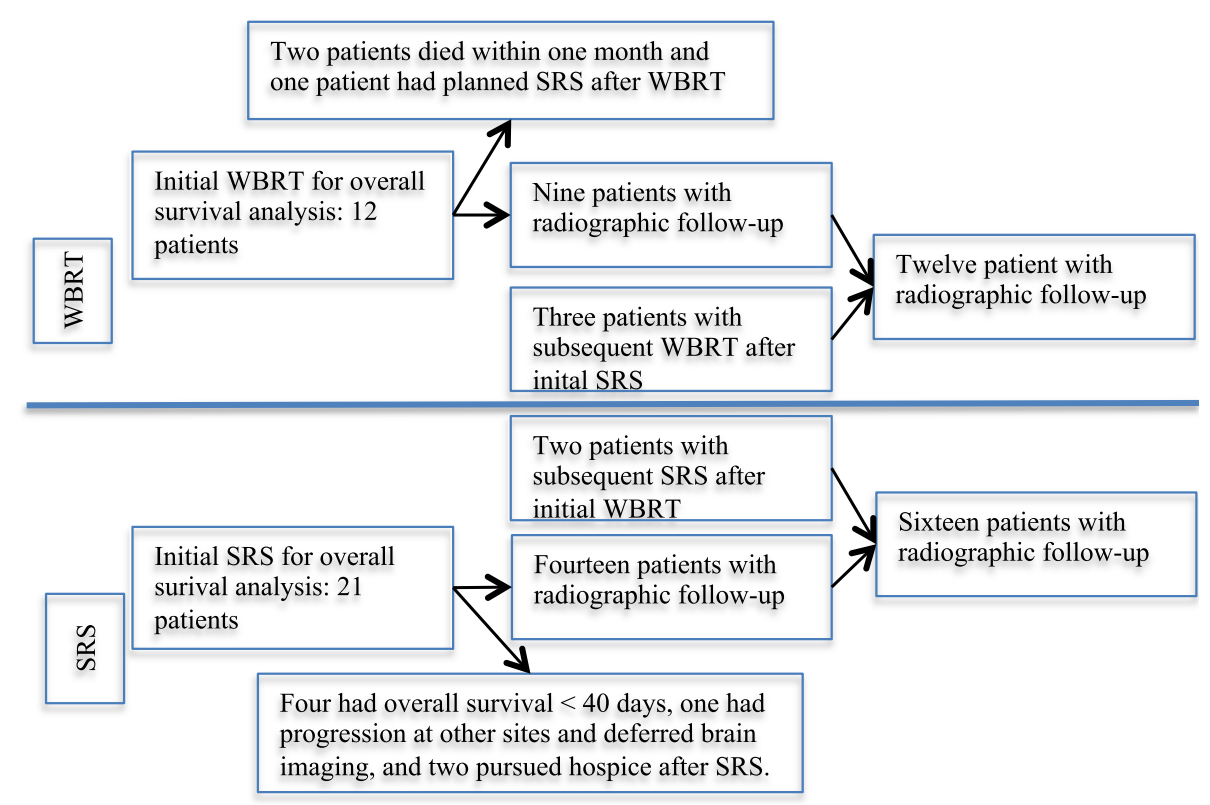

Fig. 1 Patient attrition

to the development of metastatic brain foci [5]. This is consistent with what was found in this series with $72.7 \%$ of patients presenting with liver and/or lung metastases at the time of brain metastases diagnosis. Though rare, brain metastases are an often fatal and morbid aspect of disease progression, and radiation therapy has become an accepted treatment modality for these metastatic lesions $[9,10]$. However, there is also a high competing risk of death from the significant burden of extracranial disease present in this patient population.

SRS for GI brain metastases has been documented to provide local tumor control rates of 84 to $96 \%[9,10$, 20-22]. Our results show a crude local control rate of $64.3 \%$. This is much lower than other reported rates, which may be explained by differences in prior radiation therapy, patient population, treatment modality, radiation doses, percentage adenocarcinoma histology, lesion sizes, and local failure definitions. Many studies of the clinical outcomes for GI brain metastases have included patients who previously received RT $[9,10$, 22 ]. For instance, Da Silva et al. included $55 \%$ of patients who had previously received cranial RT, and Trifiletti et al. included $36.1 \%$ of patients $[9,10]$. In contrast, Matsunaga et al. included only $5.9 \%$ of patients having previously received WBRT similar to our study which included only two lesions (7.14\%) with WBRT prior to SRS [22]. Matsunaga et al. found a crude local control rate of $91 \%$, however they defined local failure as an increase greater than $25 \%$ in maximum diameter and had a shorter median radiographic follow-up than the current series [22]. Additionally, our series treated larger lesions (mean size of $12.1 \mathrm{cc})$ than the previous studies $[9,10,22]$. Larger colorectal brain lesions have been shown to have worse local control following RT [22]. Furthermore, other studies have treated a smaller portion of adenocarcinoma histology than our current series, which treated exclusively adenocarcinoma $[9,10,22]$. Table 4 summarizes previous studies that have reported crude local control rates after RT for GI brain metastases.

The overall median survival found in this study was 5.1 months and previous studies have shown similar findings with poor outcomes following the development of GI brain metastases. A study performed by Ogawa et al. and Weinberg et al. showed a median OS of 6.7 months and 2.3 months, respectively, after the development of brain metastases from esophageal carcinoma. York et al. reported a 2.3 month median OS from gastric cancer brain metastases, and both Schoeggl et al. and Matsunaga et al. reported median survival of 6.0 months after colorectal brain metastases. This poor overall survival is worse than other published data from non-GI primaries and is indicative of the importance in identifying prognostic factors associated with these poor clinical outcomes to appropriately allocate dose escalation and/ or concurrent use of radiosensitizers [23]. Furthermore, identifying prognostic factors for poor overall survival can help us better advise patients regarding hospice management and palliative care.

Other studies analyzing GI brain metastases have found improved local control with margin dose $\geq 20$ Gy and improved survival in luminal primaries (esophagus, small bowel, stomach, colon and rectum) versus non- 


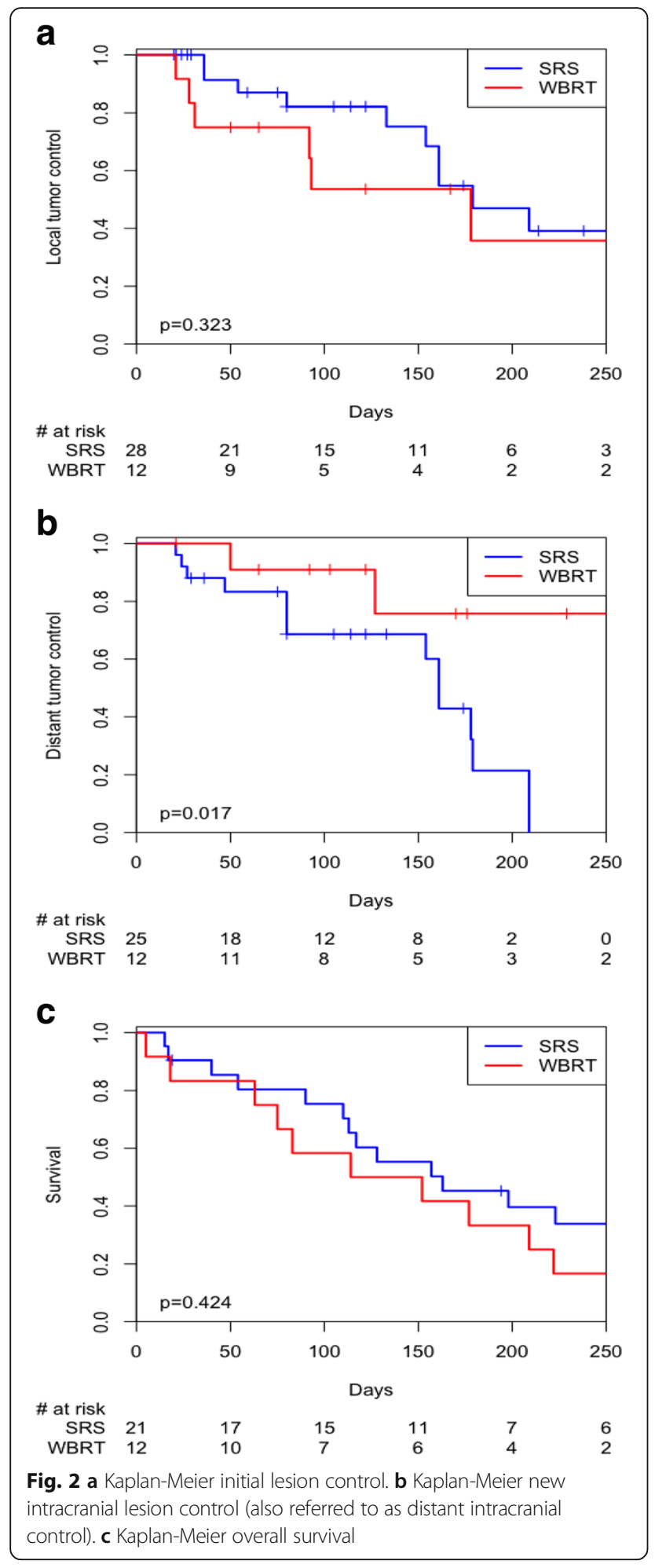

luminal primaries (pancreas and liver) [10]. A study analyzing esophageal carcinoma brain metastases found that a higher KPS was associated with an increase in survival [24]. Furthermore, a study analyzing colorectal brain metastases found unfavorable local control with larger
Table 3 Multivariate analysis for overall survival, initial site control and new site control

\begin{tabular}{lll}
\hline Characteristic & HR $(95 \% \mathrm{Cl})$ & $P$-value \\
\hline Overall survival & & \\
Age & $0.99(0.95-1.02)$ & 0.451 \\
ECOG & $1.63(0.69-3.86)$ & 0.263 \\
>2 brain metastases & $0.53(0.12-2.25)$ & 0.388 \\
Active extracranial disease & $3.02(0.62-14.79)$ & 0.173 \\
Intracranial Surgery prior to RT & $0.62(0.24-1.61)$ & 0.326 \\
Treatment WBRT & $1.39(0.45-4.22)$ & 0.566 \\
Initial site control & & \\
Total Dose & $0.69(0.31-1.55)$ & 0.372 \\
Treatment WBRT & $1.95(0.68-5.6)$ & 0.212 \\
Intracranial Surgery prior to RT & $0.47(0.16-1.36)$ & 0.164 \\
New site control & & \\
Total Dose & $3.98(1.13-14.07)$ & $0.032^{*}$ \\
Treatment WBRT & $0.05(0.01-0.35)$ & $0.002^{*}$ \\
Active extracranial disease & $11.4(1.54-84.52)$ & $0.017^{*}$ \\
\hline
\end{tabular}

Abbreviations: $\mathrm{Cl}$ confidence interval, $H R$ hazard ratio, ECOG Eastern

Cooperative Oncology group

Statistically significant results indicated by *

tumor volume and lower margin dose, while poor OS was associated with lower KPS and the presence of extracranial metastases [22]. Our study did not identify significant differences on UVA and MVA in the variables analyzed for overall survival and local control. Prior studies have demonstrated improved local control with SRS as compared to whole brain $[25,26]$; this study showed a trend towards improved local control with SRS, but did not attain statistical significance possibly due to the short radiographic follow-up periods and the small patient cohort.

Radiosensitizers are being studied for brain metastases for a variety of tumor types to improve intracranial disease control rates. Gadolinium-based nanoparticles and BRAF inhibitors concurrent with RT are being explored for melanoma brain metastases [27, 28]. Small tyrosine kinase inhibitors, small molecule HER2 targeting agents, temozolomide, and topotecan are being investigated with breast cancer [29, 30]. Furthermore in non-small cell lung cancer brain metastases, cisplatin and pemetrexed with concurrent cranial RT is actively being investigated [31]. For metastatic GI malignancies, there has been promising research done into radiosensitization with irinotecan, oxaliplatin, and biological agents for noncranial sites [32-34]. Drawing upon this research, there is potential for concurrent novel therapies to improve the efficacy of cranial irradiation [32-35]. While dose escalation has been shown to improve tumor control in radioresistant histologies, large tumor size would limit this strategy for many cases $[15,36]$. 


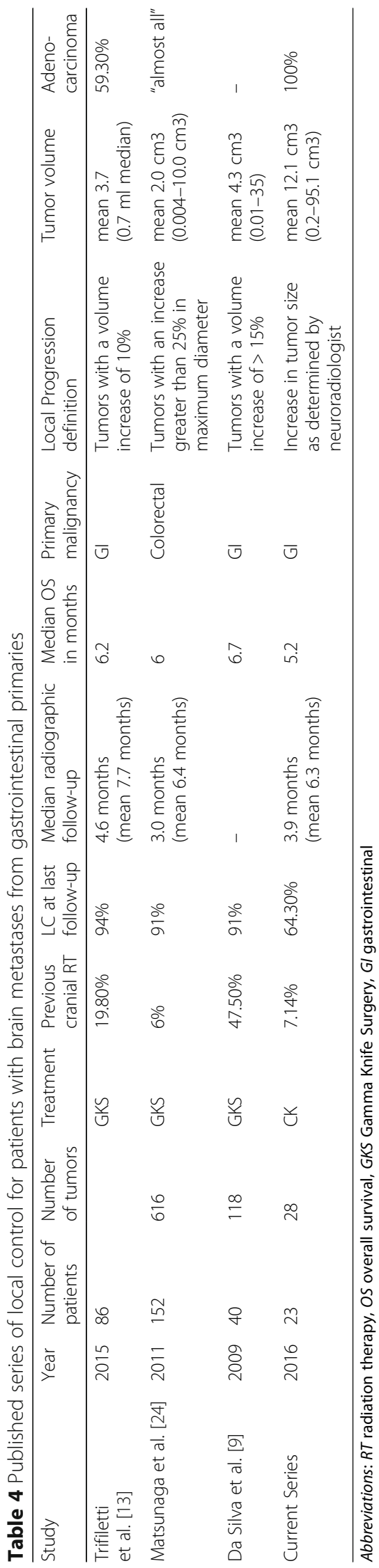


The role of WBRT and SRS in the management of brain metastases is evolving in the era of new systemic therapies [37]. In our study, overall survival was not significantly different between patients who received upfront SRS versus WBRT, however we did observe higher distant brain failure rates with SRS alone. Additionally, the association with ECD suggests tumor intracranial seeding as an important factor. A study by Aoyama et al. found no improvement in survival for the use of WBRT plus SRS, but did find the rate of intracranial relapse to be increased in those who received SRS alone similar to our series [25]. Sneed et al. also found no difference in survival when comparing SRS vs. WBRT and SRS [23]. A study by Chang et al. demonstrated an increased decline in learning and memory function in patients treated with SRS and WBRT vs. SRS, and an improved 4-month OS in the SRS alone group; however, the local and distant control was improved in the SRS plus WBRT group [14]. It is important to note that these studies were analyzing brain metastasis in general and not brain metastases from GI primaries as in the present study. Current research is exploring the utilization of SRS for greater than five brain metastases where historically WBRT was the preferred treatment modality. Yamamoto et al. demonstrated promising results when comparing patients with two to four brain metastases versus five to ten brain metastases treated with SRS and found non-inferiority in overall survival [15]. Compared to WBRT, SRS is associated with a shorter treatment duration, higher BED, and reduced neurocognitive deficits $[14,15]$.

Limitations of this study include its retrospective nature and the small sample size due to the relative rarity of this patient population. Additionally, given the short radiographic follow-up and the inclusion of cavitary lesions, it is often challenging to differentiate between radionecrosis and progression. Nevertheless, due to the relatively poor prognosis associated with brain metastases from GI primaries, future research should continue to assess prognostic and predictive factors, optimal dose fractionation schedules, treatment paradigms, and adverse effects. Future research should also attempt to discern the effect of radiation therapy on patient reported outcomes and quality of life endpoints. As survival improves for metastatic GI cancer with advances in systemic therapies, the incidence of brain metastasis will likely rise necessitating further improvement in treatments.

\section{Conclusion}

Survival and intracranial disease control are poor following RT for brain metastases from GI primaries. In this small series, outcomes are worse than published series for other primary malignancies metastatic to the brain and further research into methods of local control improvement is warranted. The poor overall survival and local control could potentially be due to (1) radioresistant biology of GI malignancies, and (2) brain metastases in GI malignancies are generally a late event with the majority of patients presenting with diffuse active extracranial disease. The association found between extracranial disease and the development of new intracranial disease should be further explored. Future studies should elucidate the utility of dose escalation and/or radiosensitization in this patient population.

\section{Abbreviations \\ BED: Biologically equivalent dose; Cl: Confidence interval; CT: Computed tomography; ECOG: Eastern Cooperative Oncology Group; \\ GI: Gastrointestinal; GKS: Gamma Knife Surgery; GTV: Gross Tumor Volume; HR: Hazard ratio; KPS: Karnofsky Performance Status; LINAC: Linear accelerator; MR: Magnetic resonance; MRI: Magnetic resonance imaging; MVA: Multivariate analysis; N: Number; PET: Positron emission tomography; RT: Radiation therapy; SRS: Stereotactic radiosurgery; UVA: Univariate analysis; WBRT: Whole brain radiation therapy}

\section{Acknowledgements}

The authors would like to acknowledge the assistance of the staff at the Department of Radiation Oncology at Georgetown University for their assistance in obtaining necessary charts, and of Huiqi Zhuang for her assistance with data analysis.

Funding

This study was not supported by outside funding.

\section{Availability of data and materials}

The datasets generated during and/or analyzed during the current study are not publicly available due patient confidentiality but are available from the corresponding author on reasonable request.

\section{Authors' contributions}

All authors contributed to study concept, design, and/or acquisition of data. SMS and KU completed the data collection. SMS and LC contributed to the data analysis. SMS and JWL contributed to drafting the manuscript. All authors contributed to revising and giving final approval to the manuscript. All authors agree to be accountable for all aspects of the work including its accuracy and integrity.

\section{Competing interests}

The authors declare that they have no competing interests.

\section{Consent for publication}

Not applicable.

\section{Ethics approval and consent to participate}

All procedures performed in studies involving human participants were in accordance with the ethical standards of the institutional and/or national research committee and with the 1964 Helsinki declaration and its later amendments or comparable ethical standards. The local Health Research Institutional Review Board (IRB) approved this retrospective analysis of an established departmental treatment approach.

\section{Informed consent}

For this type of study, formal consent is not required.

\section{Author details}

${ }^{1}$ Georgetown University School of Medicine, Medical Dental Building, 3900 Reservoir Road, N.W, Washington DC 20057, USA. ${ }^{2}$ Department of Radiation Medicine, Lombardi Comprehensive Cancer Center, Georgetown University Hospital, Lower Level Bles, 3800 Reservoir Road, N.W, Washington DC 20007, USA. ${ }^{3}$ Department of Biostatistics, Bioinformatics, and Biomathematics, Georgetown University, 4000 Reservoir Rd., NW, Washington DC 20057, USA. 
${ }^{4}$ Department of Neurosurgery, 3800 Reservoir Rd. NW, Pasquerilla Healthcare Center Seventh Floor, Washington DC 20007, USA.

Received: 15 November 2016 Accepted: 6 February 2017 Published online: 28 February 2017

\section{References}

1. Surveillance, Epidemiology, and End Results (SEER) Program (http://www. seer.cancer.gov) Research Data (1973-2013). National Cancer Institute, DCCPS, Surveillance Research Program, Surveillance Systems Branch. Accessed 19 July 2016.

2. Floyd CE, Stirling CT, Cohn Jr I. Cancer of the colon, rectum and anus: review of 1687 cases. Ann Surg. 1966;163(6):829-37.

3. Salvati $\mathrm{M}$, et al. Solitary cerebral metastases from intestinal carcinoma. Acta Neurochir (Wien). 1995;133(3-4):181-3.

4. Weiss $L$, et al. Haematogenous metastatic patterns in colonic carcinoma: an analysis of 1541 necropsies. J Pathol. 1986;150(3):195-203.

5. Qiu M, et al. Pattern of distant metastases in colorectal cancer: a SEER based study. Oncotarget. 2015;6(36):38658-66.

6. Bartelt $\mathrm{S}$, et al. Patients with brain metastases from gastrointestinal tract cancer treated with whole brain radiation therapy: prognostic factors and survival. World J Gastroenterol. 2004;10(22):3345-8

7. Cascino TL, et al. Brain metastases from colon cancer. J Neurooncol. 1983; 1(3):203-9.

8. Delattre JY, et al. Distribution of brain metastases. Arch Neurol. 1988; 45(7):741-4.

9. Da Silva AN, et al. Gamma Knife surgery for brain metastases from gastrointestinal cancer. J Neurosurg. 2009;111(3):423-30.

10. Trifiletti DM, et al. Stereotactic radiosurgery in the treatment of brain metastases from gastrointestinal primaries. J Neurooncol. 2015;124(3):439-46.

11. Go PH, et al. Gastrointestinal cancer and brain metastasis: a rare and ominous sign. Cancer. 2011;117(16):3630-40.

12. Tabouret $\mathrm{E}$, et al. Recent trends in epidemiology of brain metastases: an overview. Anticancer Res. 2012;32(11):4655-62.

13. Bezjak $A$, et al. Radiotherapy for brain metastases: defining palliative response. Radiother Oncol. 2001;61(1):71-6.

14. Chang EL, et al. Neurocognition in patients with brain metastases treated with radiosurgery or radiosurgery plus whole-brain irradiation: a randomised controlled trial. Lancet Oncol. 2009;10(11):1037-44.

15. Yamamoto M, et al. Stereotactic radiosurgery for patients with multiple brain metastases (JLGK0901): a multi-institutional prospective observational study. Lancet Oncol. 2014;15(4):387-95.

16. Holt $D E$, et al. Tumor bed radiosurgery following resection and prior stereotactic radiosurgery for locally persistent brain metastasis. Front Oncol. 2015:5:84.

17. Maranzano E, et al. LINAC- Based adiosurgery for melanoma, sarcoma and renal cell carcinoma brain metastases. J Neurosurg Sci, 2016. [Epub ahead of print].

18. Son $\mathrm{CH}$, et al. Outcomes after whole brain reirradiation in patients with brain metastases. Int J Radiat Oncol Biol Phys. 2012;82(2):e167-72.

19. Collins SP, et al. Cyberknife radiosurgery in the treatment of complex skull base tumors: analysis of treatment planning parameters. Radiat Oncol. 2006:1:46

20. Schoeggl A, et al. Stereotactic radiosurgery for brain metastases from colorectal cancer. Int J Colorectal Dis. 2002:17(3):150-5.

21. Hasegawa $T$, et al. Stereotactic radiosurgery for brain metastases from gastrointestinal tract cancer. Surg Neurol. 2003;60(6):506-14. discussion 514-5.

22. Matsunaga $S$, et al. Gamma Knife surgery for brain metastases from colorectal cancer. Clinical article. J Neurosurg. 2011;114(3):782-9.

23. Sneed PK, et al. A multi-institutional review of radiosurgery alone vs. radiosurgery with whole brain radiotherapy as the initial management of brain metastases. Int J Radiat Oncol Biol Phys. 2002;53(3):519-26.

24. Bowden G, et al. Gamma knife radiosurgery for management of cerebral metastases from esophageal carcinoma. J Neurooncol. 2014;1 18(1):141-6.

25. Aoyama $\mathrm{H}$, et al. Stereotactic radiosurgery plus whole-brain radiation therapy vs stereotactic radiosurgery alone for treatment of brain metastases: a randomized controlled trial. JAMA. 2006;295(21):2483-91.

26. Scheitler-Ring $\mathrm{K}$, et al. Radiosurgery to the postoperative tumor Bed for metastatic carcinoma versus whole brain radiation after surgery. Cureus. 2016;8(11):e885.
27. Kotb S, et al. Gadolinium-based nanoparticles and radiation therapy for multiple brain melanoma metastases: proof of concept before phase I trial. Theranostics. 2016;6(3):418-27.

28. Gaudy-Marqueste $C$, et al. On demand gamma-knife strategy can be safely combined with BRAF inhibitors for the treatment of melanoma brain metastases. Ann Oncol. 2014;25(10):2086-91.

29. Cao Kl, Kirova YM. Radiotherapy plus concomitant systemic therapies for patients with brain metastases from breast cancer. Cancer Radiother. 2014; 18(3):235-42. quiz 246, 249.

30. Koo T, Kim IA. Brain metastasis in human epidermal growth factor receptor 2-positive breast cancer: from biology to treatment. Radiat Oncol J. 2016;34(1):1-9.

31. Dinglin $X X$, et al. Pemetrexed and cisplatin combination with concurrent whole brain radiotherapy in patients with brain metastases of lung adenocarcinoma: a single-arm phase II clinical trial. J Neurooncol. 2013: 112(3):461-6.

32. Greenhalgh TA, Dearman C, Sharma RA. Combination of Novel Agents with Radiotherapy to Treat Rectal Cancer. Clin Oncol (R Coll Radiol). 2016;28(2): 116-39.

33. Kirstein MM, et al. Targeted therapies in metastatic colorectal cancer: a systematic review and assessment of currently available data. Oncologist. 2014:19(11):1156-68

34. Macedo LT, da Costa Lima AB, Sasse AD. Addition of bevacizumab to firstline chemotherapy in advanced colorectal cancer: a systematic review and meta-analysis, with emphasis on chemotherapy subgroups. BMC Cancer. 2012;12:89.

35. Mollica A, et al. Delivery methods of camptothecin and its hydrosoluble analogue irinotecan for treatment of colorectal cancer. Curr Drug Deliv. 2012;9(2):122-31.

36. Meyners T, et al. Prognostic factors for outcomes after whole-brain irradiation of brain metastases from relatively radioresistant tumors: a retrospective analysis. BMC Cancer. 2010;10:582.

37. Shen CJ, Lim M, Kleinberg LR. Controversies in the Therapy of Brain Metastases: Shifting Paradigms in an Era of Effective Systemic Therapy and Longer-Term Survivorship. Curr Treat Options Oncol. 2016;17(9):46.

\section{Submit your next manuscript to BioMed Central and we will help you at every step:}

- We accept pre-submission inquiries

- Our selector tool helps you to find the most relevant journal

- We provide round the clock customer support

- Convenient online submission

- Thorough peer review

- Inclusion in PubMed and all major indexing services

- Maximum visibility for your research

Submit your manuscript at www.biomedcentral.com/submit
) Biomed Central 